\title{
Grain size gradient length scale in ballistic properties optimization of functionally graded nanocrystalline steel plates
}

\author{
Antoine Jérusalem, ${ }^{\mathrm{a}, *}$ William Dickson, ${ }^{\mathrm{b}}$ Maria Jesús Pérez-Martín, ${ }^{\mathrm{c}}$ Ming Dao, ${ }^{\mathrm{b}}$ \\ Jian $\mathrm{Lu}^{\mathrm{d}}$ and Francisco Gálvez ${ }^{\mathrm{c}}$
}

The last few years have highlighted the existence of two relevant length scales in the quest to ultrahigh-strength polycrystalline metals. Whereas the microstructural length scale - e.g. grain or twin size - has mainly be linked to the well-established Hall-Petch relationship, the sample length scale - e.g. nanopillar size - has also proven to be at least as relevant, especially in microscale structures. In this letter, a series of ballistic tests on functionally graded nanocrystalline plates are used as a basis for the justification of a "grain size gradient length scale" as an additional ballistic properties optimization parameter.

Recent research works fueled by the recent advances in fabrication processes have emphasized the need to better understand naturally or artificially made size effects in materials in order to enhance their properties [1]. Material strength, more particularly, has been found to be microstructurally linked to two length scales [2]. The microstructural length scale - or intrinsic length scale - is related to the size of the material building block micro- or nanostructures. In polycrystalline metals, the most common examples are the grain and/or twin sizes which, refined to the nanometer range $(<100 \mathrm{~nm})$, effectively reduce the dislocations mobility, and thus achieve very high yield strength and surface hardness - as predicted by the Hall-Petch equation $[3,4]$. The second length scale - or extrinsic length scale - is related to the sample size. In metals, the typical example is the ultrahigh strength of monocrystalline nanopillars [5], directly related to the size-dependent scarcity of initial dislocations (starved first before further nucleation [6]).

A recent experimental-numerical campaign has highlighted the potential of nanocrystals and nanotwinned ultrafine crystals steel for ballistic protection systems [7]. In this reference, hybridization with a carbon fiber-epoxy composite layer was proposed as a way to improve the nanocrystalline brittleness without dramatically increasing the overall weight. Ultimately, nanocrystalline and nanotwinned ultrafine crystals exhibited a lower ballistic energy absorption than coarse-grained steel, but at equal ballistic limit or weight prior to penetration, deformation in the impact direction was found to be smaller by nearly $40 \%$ [7].

Surface mechanical attrition treatment (SMAT), the process used for the fabrication of these nanocrystalline and nanotwinned ultrafine crystalline plate, generates a gradient of ultrafine crystal grain sizes $([8,7])$. Subsequent coating with a nitriding layer further refines the grains on the top layer to the nanoscale [9,7]. Both processes are nevertheless localized at the surface, leading to a gradient of grain size ranging from the targeted nanocrystals to 
the coarse grains of the original stainless steel plates. In Ref. [7], both faces of the plates were subjected to the treatment in order to maximize plate homogeneity.

In this letter, an attempt to directly leverage the higher ductility of the coarse-grained steel plates as a support to their nanocrystalline counterparts is proposed. To this end, the SMAT-induced grain size gradient is utilized as a means to further optimize the ballistic energy absorption of the plates. The results of Ref. [7] would indicate that by impacting the coarse-grained side first, the brittle behaviour of the nanocrystalline layer could be avoided a priori by first spreading the shock in a similar way as what was achieved by the previously used carbon-epoxy composite layer. However, the relative contribution of this grain size gradient spread with respect to the finest grain size (the intrinsic length scale) or the sample thickness (the extrinsic length scale) is not clear. As a consequence, different sample thicknesses are considered here. Because of the increased ductility of nanotwinned ultrafine crystals with respect to nanocrystals [10], for the same ballistic behavior and similar strength [7], the former were chosen for the proposed study. AISI 304 stainless steel sheets were commercially acquired with thickness of 1,2 and $4 \mathrm{~mm}$. They were cut into $50 \mathrm{~mm} \times 70 \mathrm{~mm}$ plates for SMAT, which was carried out on one face for $5 \mathrm{~min}$, using $3 \mathrm{~mm}$ diameter stainless steels balls (see Ref. [7] for more details on the treatment).

Tensile samples were first obtained by wire cutting, with a gauge dimension of $34 \mathrm{~mm}$ length and $6 \mathrm{~mm}$ width. Tensile tests were carried out on an MTS-Alliance RT/50 machine, with a velocity of $1.5 \mathrm{~mm} \mathrm{~min}^{-1}$. Three samples for each thickness were used for the average response of the plates (with very little standard deviation). The results are given in Fig. 1 and Table 1.

The results exhibit higher yield stress and tensile strength but lower ductility after SMAT treatment for all three thicknesses. For the non-treated plates, the $2 \mathrm{~mm}$ thickness case presents a slightly higher yield stress but a slightly lower tensile strength and ductility than the $1 \mathrm{~mm}$ thickness case. The $4 \mathrm{~mm}$ case differs

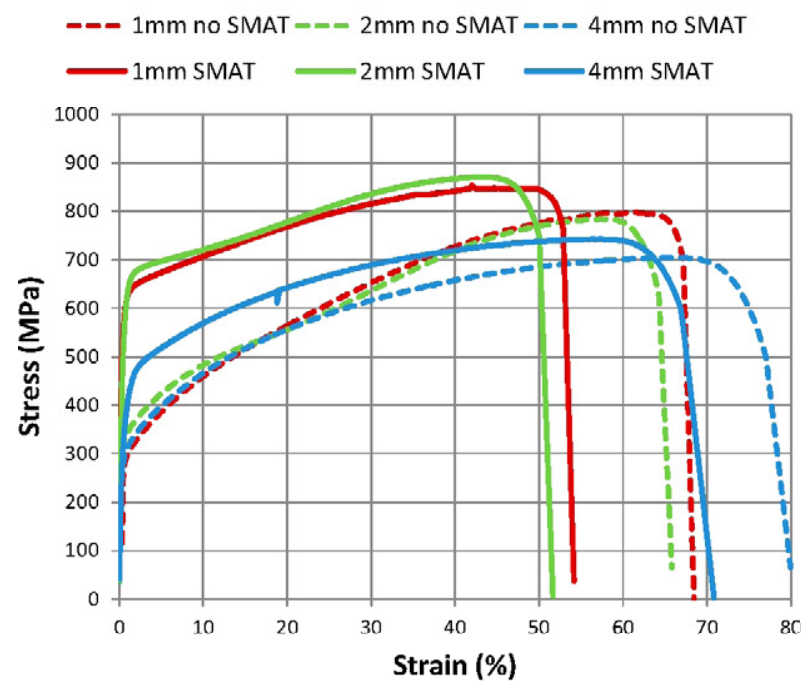

Figure 1. Averaged tensile curves of AISI 304 stainless steel plates with thicknesses of 1,2 and $4 \mathrm{~mm}$ as received and after one-face SMAT. from both cases with significantly lower tensile strength and higher ductility. For the treated plates, the $2 \mathrm{~mm}$ thickness case also presents a slightly higher yield stress and lower ducility than the $1 \mathrm{~mm}$ thickness case. Finally, similarly to the non-treated case but more dramatically, the treated $4 \mathrm{~mm}$ thickness case presents a lower yield stress and tensile strength but a much larger ductility than the other thicknesses.

The large difference between the $4 \mathrm{~mm}$ cases and the 1 and $2 \mathrm{~mm}$ cases is either due to a geometrical effect of the section during necking or a processing artifact. In order to identify which one of the two possibilities applies, a series of nanoindentation tests with a Nanoindenter XP from MTS Systems Corporation (Oak Ridge, TN, USA) was done at room temperature across the thickness of each plate. Because the SMAT treatment is superficial, only the treated plates were nanoindented (the non-treated hardnesses can be extracted from the coarse grain part of the treated plates). The samples were cross-sectioned using a diamond saw, and polished with 2400 grit $\mathrm{SiC}$ paper and diamond slurry to a $1 \mu \mathrm{m}$ finish. The depth-sensing indentation tests were carried out using a Berkovich indenter with a nominal edge radius of $500 \mathrm{~nm}$. The system's load and displacement resolutions are of $50 \mathrm{nN}$ and $0.01 \mathrm{~nm}$, respectively. The row of indentations across the thickness was done with a maximum depth of indentation of $400 \mathrm{~nm}$ and at a strain rate of $0.05 s^{-1}$. The distance between the indentations was set to $20 \mu \mathrm{m}$. Finally, the Oliver and Pharr method [11] was used to obtain the elastic modulus and the hardness at each location of indentation (see Fig. 2).

The similar Young's moduli and hardnesses in the non-treated region seem to indicate that the differences observed for the $4 \mathrm{~mm}$ cases in Fig. 1 are due to geometrical effects at the onset of necking. The Young's modulus remains the same in all cases and the hardnesses increase continuously in the last $\sim 0.5 \mathrm{~mm}$ (within the treated zone) in all three cases, increasing up to $50 \%$ for the finest grains. Note that this behavior is in close qualitative agreement with recent work on SMAT-treated ultrafine-grained titanium [12] and confirms the very attractive properties of nanograined coated metals as discussed thoroughly in Ref. [13].

A series of gas gun ballistic tests were then done with pressurized air and helium, and $5.55 \mathrm{~mm}$ diameter stainless steel spherical projectiles. All projectiles were placed in (and shot along with) a $7 \mathrm{~mm}$ diameter steel plate sabot from which they separated before reaching the plates. Compressed air allowed for projectile velocities ranging from 270 to $440 \mathrm{~m} \mathrm{~s}^{-1}$, and helium from 440 to $800 \mathrm{~m} \mathrm{~s}^{-1}$. A Phantom high-speed camera, recording at 80,000 frames $s^{-1}$, was placed so as to capture the path of the bullet before and after impact. Two 144 watt external light sources illuminated the sample during recording in order to provide sufficient exposure for the camera. The velocities before and after impact were then calculated from the analysis of the recording. Fig. 3 shows four different estimations of the absorbed energy per areal density at the ballistic limit for the three thicknesses and for the three cases: no SMAT, SMAT layer facing towards the gun and SMAT layer facing away from the gun. The four estimations are provided by: 
Table 1. Mechanical properties of the AISI 304 stainless steel plates with thicknesses of 1,2 and 4 mm as received and after SMAT.

\begin{tabular}{llll}
\hline Sample $(\mathrm{mm})$ & Yield stress $(\mathrm{MPa})$ & Tensile strength $(\mathrm{MPa})$ & Elongation $(\%)$ \\
\hline 1-no SMAT & $290.54 \pm 3.12$ & $795.43 \pm 3.93$ & $63.23 \pm 0.12$ \\
1-SMAT & $590.4 \pm 11.89$ & $857.13 \pm 11.91$ & $49.07 \pm 0.57$ \\
2-no SMAT & $314.53 \pm 3.53$ & $782.13 \pm 1.27$ & $60.7 \pm 0.36$ \\
2-SMAT & $598.75 \pm 13.7$ & $866.17 \pm 5.78$ & $46.1 \pm 1.5$ \\
4-no SMAT & $276.82 \pm 9.95$ & $702.2 \pm 2.57$ & $73.37 \pm 1.03$ \\
4-SMAT & $415.46 \pm 3.31$ & $740.47 \pm 3.26$ \\
\hline
\end{tabular}

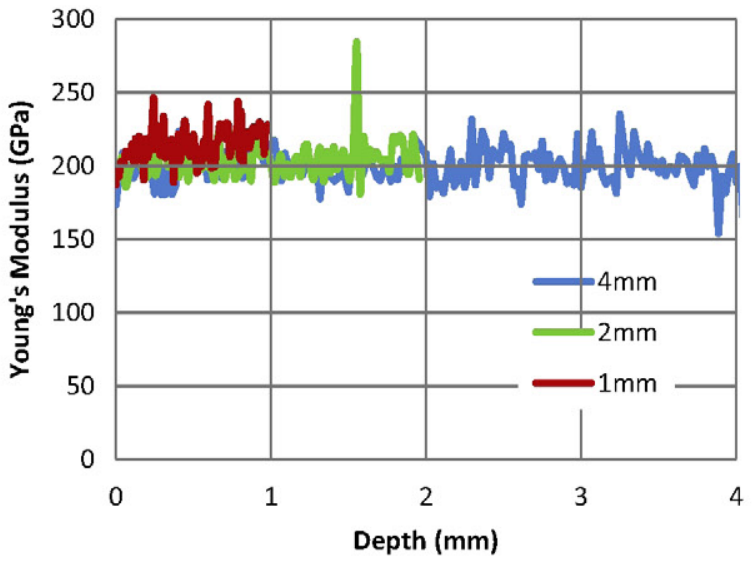

(a)

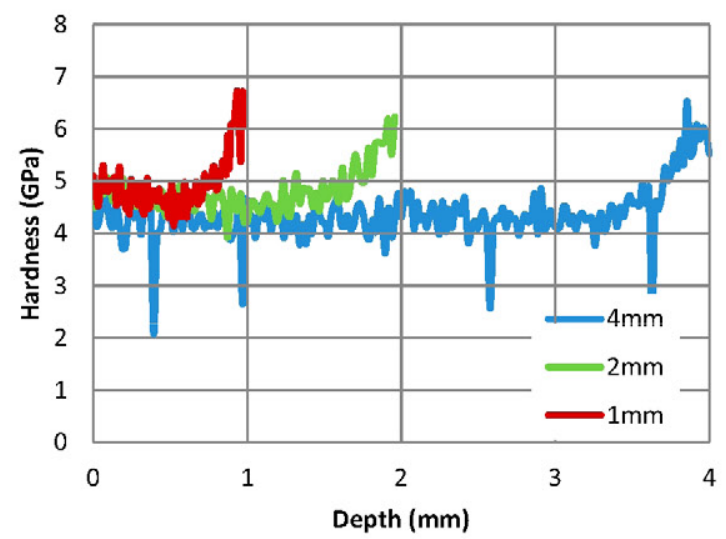

(b)

Figure 2. Young's modulus and hardness across the thicknesses (1, 2 and $4 \mathrm{~mm}$ ) of the treated plates; the measurements start on the nontreated side.

(i) the energy corresponding to the highest projectile velocity not perforating the plate, and the energies corresponding to the first velocity for which the projectile perforated the plate (ii) substracting the energies of the outgoing projectile and plug, (iii) substracting the energy of the outgoing projectile only and (iv) accounting only for the incoming velocity. Note that, in some cases, one or more of the energies could not be calculated (when outgoing projectiles and/or plugs could not be calculated, e.g. because of fragmentation, out-of-plane trajectory, plug not found). Case (i) can thus be considered as a lower bound, whereas cases (ii), (iii) and (iv) could a priori be thought as upper bounds, increasing in that order.

In some cases (i.e. $2 \mathrm{~mm}$, "no SMAT"), the three "upper bounds", (ii), (iii) and (iv), increase above the lower bound (i), as expected, but for others (i.e. $2 \mathrm{~mm}$,
"SMAT back"), one or more of these "upper bounds" exhibit a lower energy than the lower bound (i). However, for all cases, the absorbed energy subsequently increases with increasing incoming projectile velocity (not shown), which could indicate an erroneous measure of either the outgoing projectile velocity or the plug velocity (or both) for those "upper bounds" that are lower than case (i). It is also possible that the ductile fracture (dishing) observed at the ballistic limit and quickly transitioning into brittle failure (plugging) [7] could lead to an immediate drop in the absorbed energy, thus explaining the apparent contradiction. Note that the fact that the areal density decreases slightly between a non-treated plate and its treated counterpart (because of thicknesses being slightly reduced during treatment) also increases the absorbed energy per areal density slightly. Finally, the $1 \mathrm{~mm}$ "SMAT back" case ballistic limit was found to be lower than the gun lowest accessible velocity, and no lower bound could be calculated. To sum up, the following tendencies for all three thicknesses can be identified: the three configurations "no SMAT", "SMAT front" and "SMAT back" exhibit, in this order, a decreasing absorbed energy per areal density at ballistic limit for the $1 \mathrm{~mm}$ case, an increasing energy for the $2 \mathrm{~mm}$ case, and a shallow "U-shaped" behavior with a higher right branch for the $4 \mathrm{~mm}$ case. The ballistic limits follow the same trends.

Finally, the deformation at the ballistic limit was estimated by placing the plug on top of the deformed plate for the first non-zero projectile outgoing velocity cases and measuring the distance between an edge of the plate and the tip of the plug using their silhouettes with a Nikon Profile Projector. When the lower bound velocity was estimated to be close enough to the ballistic limit, the corresponding measurement (with only dishing failure) was used instead. When more than one test was available, the results were averaged. The results are shown in Fig. 4, with the error bars estimated from all possible measurement errors.

For all thicknesses, the deformation exhibits a smaller deformation for the "SMAT front" case. The "SMAT back" deformation is less than the non-treated counterpart for the $2 \mathrm{~mm}$ case but is larger in the $4 \mathrm{~mm}$ case. Finally, the $2 \mathrm{~mm}$ cases present smaller deformations than the two other thicknesses.

Gathering all these findings together, the results for "no SMAT" exhibit a relatively constant absorbed energy per areal density $\left(\sim 4.5 J^{2} \mathrm{~m}^{-1} \mathrm{~kg}^{-1}\right)$ for all three thicknesses. Positioning the plates with the treated side away from the gun clearly shows a marked improvement over the other configurations for the 2 and $4 \mathrm{~mm}$ thickness cases (higher absorbed energy and higher ballistic limit). The deformation is, however, less marked than 


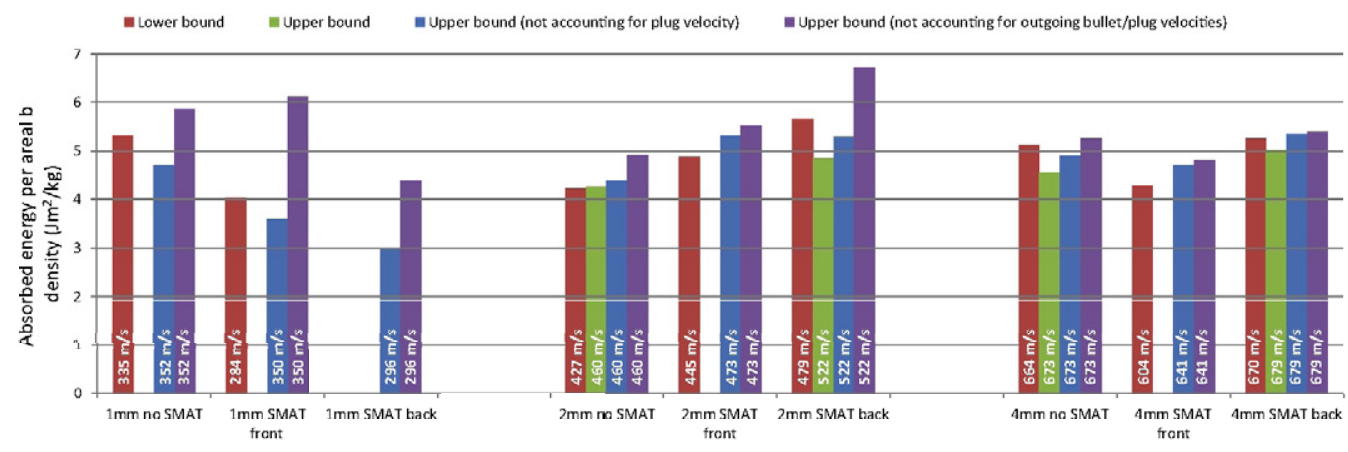

Figure 3. Absorbed energy per areal density for the three thicknesses and for the three cases: no SMAT, SMAT layer facing towards gun and SMAT layer facing away from the gun; for each configuration the provided energies are: (i) the energy corresponding to the highest projectile velocity not perforating the plate, and the energies corresponding to the first velocity for which the projectile perforated the plate (ii) substracting both energies of the outgoing projectile and the plug, (iii) substracting only the energy of the outgoing projectile and (iv) accounting only for the incoming velocity. The corresponding projectile incoming velocities are provided at the bottom of the bars.

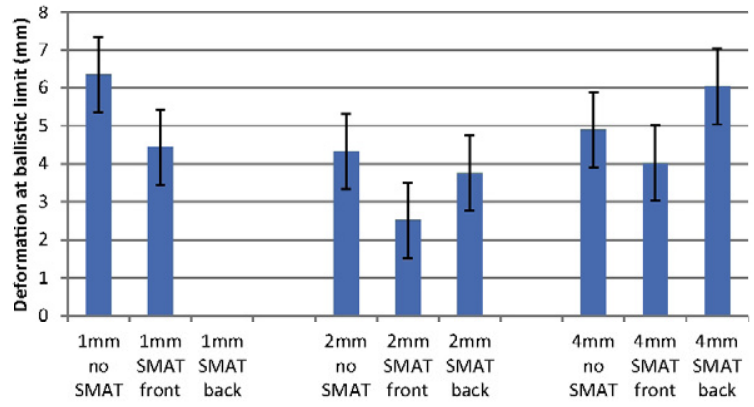

Figure 4. Deformations at ballistic limit for 1,2 and $4 \mathrm{~mm}$ thicknesses for the three configurations: as received, and after one-face SMAT facing gun and away from the gun ( $1 \mathrm{~mm}$ "SMAT back" ballistic limit was not reached as it was lower than the gun's lowest accessible velocity).

for the non-treated plate only in the $2 \mathrm{~mm}$ case. Conversely, placing the treated face towards the gun consistently leads to a smaller deformation, but exhibits a lower absorbed energy and ballistic limit than the other configurations in the $4 \mathrm{~mm}$ case while performing better than the non-treated case in the $2 \mathrm{~mm}$ case.

The intrinsic length scale (i.e. the finest grain size) is the same in all configurations. The extrinsic length scale (i.e. the thickness) by itself is not sufficient to account for the change in energy - as shown by the non-treated cases. Similarly, the grain size gradient spread also remains constant in all treated configurations $(\sim 0.5 \mathrm{~mm})$. As a consequence, we propose that the simultaneous consideration of all three length scales is necessary for any optimization problem, and that their combination can be used as a new set of optimization parameters. Following such optimization, a minimum deformation for a maximum higher ballistic limit and absorbed energy can be reached with treated plates. For the specific problem tackled in this letter, the ratio between the energy and the deformation could potentially be used as the function to maximize. In this case, the " 2 mm SMAT front" is actually the best of all cases and leads to the conclusion that placing the treated face towards the gun would actually be "better" than placing it away from it (i.e. the opposite conclusion to the one made in Ref. [7] when using carbon-epoxy composite layers). Additionally, there must be an optimum thickness between 1 and $4 \mathrm{~mm}$ (and probably close to $2 \mathrm{~mm}$ ) for which the function is fully maximized.
More generally, the optimization of any given functionally graded polycrystalline metallic plate for ballistic performance could thus be done by considering both the intrinsic and extrinsic length scales defined above, along with this new grain size gradient length scale. Note finally that the length scale related to the size of the projectile has been ignored throughout this analysis but will have to be taken into account as an additional parameter for any study using different projectile diameters.

Future work will encompass a comprehensive modeling campaign aimed at designing the ballistic performance of these functionally graded nanocrystalline steel plates against these three optimization parameters.

W.D. acknowledges funding through the http://web.mit.edu/misti/mit-spain/MIT Spain program. M.J.P. and F.G. also gratefully acknowledge support from the Spanish Government (BIA2011-24445 project). M.D. acknowledges partial support from Singapore MIT Alliance (SMA).

[1] L. Valdevit, A. Jacobsen, J. Greer, W. Carter, J. Am. Ceram. Soc. 94 (2011) 15-34.

[2] J. Greer, J. De Hosson, Prog. Mater Sci. 56 (2011) 654 724.

[3] S. Yip, Nature 391 (1998) 532-533.

[4] L. Lu, X. Chen, X. Huang, K. Lu, Science 323 (2003) 607-610.

[5] J. Greer, W. Oliver, W. Nix, Acta Mater. 53 (2005) 18211830.

[6] A. Jérusalem, A. Fernández, A. Kunz, J. Greer, Scr. Mater. 66 (2012) 93-96.

[7] J.F. Vicente, Y. Zhang, M. Dao, J. Lu, F. Gálvez, A. Jérusalem, Acta Mater. 60 (2012) 1353-1367.

[8] H. Zhang, Z. Hei, G. Liu, J. Lu, K. Lu, Acta Mater. 51 (2003) 1871-1881.

[9] Y. Lin, J. Lu, L. Wang, T. Xu, Q. Xue, Acta Mater. 54 (2006) 5599-5605.

[10] A. Jérusalem, M. Dao, S. Suresh, R. Radovitzky, Acta Mater. 56 (2008) 4647-4657.

[11] W. Oliver, G. Pharr, J. Mater. Res. 7 (1992) 1564-1583.

[12] D. Yang, P. Cizek, D. Fabijanic, P. Hodgson, Materials Science and Engineering A (In press).

[13] T. Fang, W. Li, N. Tao, K. Lu, Science 331 (2011) 15871590. 